\title{
Vertragswettbewerb zwischen Krankenkassen in der
}

\section{integrierten Versorgung}

"Die integrierte Versorgung hat sich gut entwickelt." ${ }^{1}$ So lautet das Fazit des Gesetzgebers über die bisherigen Anstrengungen der Beteiligten um die Etablierung integrierter Versorgungsmodelle in den Materialien zum Gesetz zur Stärkung des Wettbewerbs in der Gesetzlichen Krankenversicherung (GKV-WSG) ${ }^{2}$.

"Diese Entwicklung ist zu unterstützen." formuliert der Gesetzgeber weiter quasi als Ziel oder als Programm für die Änderungen, die dann durch das GKV-WSG an den Vorschriften zur integrierten Versorgung vorgenommen werden.

Im folgenden Beitrag soll der Frage nachgegangen werden, inwieweit die Änderungen an den Regelungen in Bezug auf die integrierte Versorgung in den jüngsten Reformen dem Anspruch des Gesetzgebers tatsächlich genügen, die Weiterentwicklung der integrierten Versorgung zu unterstützen. Ein besonderer Augenmerk soll darauf gerichtet werden, ob die Änderungen den Vertragswettbewerb fördern.

\section{Jutta Kaempfe}

Man wird zu dem Ergebnis kommen müssen, dass die Neuerungen diesem Anspruch im Wesentlichen genügen. Ausnahmen bestätigen allerdings auch hier die Regel. Durch diese grundsätzlich positive Einschätzung hebt sich die Beurteilung der rechtlichen Änderungen im Bereich der integrierten Versorgung von den meisten anderen Änderungen des GKV-WSG ab.

\section{Thema und Gang des Beitrags}

1.1 Das Thema des folgenden Beitrags ist in dreierlei Hinsicht eingegrenzt: Gegenstand ist der Status Quo der integrierten Versorgung in rechtlicher Hinsicht nach den jüngsten Reformen des Gesundheitswesens. Weder die Darstellung, wie sich die Vorschriften über die integrierte Versorgung bis ins Jahr 2007 entwickelt haben, noch wie sie sinnvollerweise mittelfristig fortzuentwickeln sind, ist also beabsichtigt. Untersucht werden können hier ferner nur die Rechtsvorschriften, Vergleiche verschiedener wirtschaftswissenschaftlicher Modelle o.ä. werden nicht vorgenommen.

1.2 Nach einer kurzen Einführung zum Vertragswettbewerb im Allgemeinen soll auf den Vertragswettbewerb in der integrierten Versorgung eingegangen werden. Zuerst wird das Verhältnis der Krankenkassen zu ihren Vertragspartnern und dann das Verhält-

Dr. Jutta Kaempfe, Stabsbereich Recht, AOK-Bundesverband, Bonn nis der Krankenkassen zu den Versicherten beleuchtet. Nach der Nennung einiger weiterer Einflussfaktoren für das Gelingen von Vertragswettbewerb sollen die Neuregelungen zur integrierten Versorgung einer ersten zusammenfassenden Bewertung unterzogen werden.

\section{Vertragswettbewerb allgemein}

\subsection{Das Konzept des Vertragswettbewerbs}

Grundlage der Ausführungen zum Vertragswettbewerb allgemein sind zwei Gutachten zum Vertragswettbewerb in der gesetzlichen Krankenversicherung bzw. zu dessen Weiterentwicklung, die im Auftrag des AOK-Bundesverbandes in den Jahren 2003 bzw. 2006 erstellt wurden. ${ }^{3}$

Die Gutachter kommen zu dem Ergebnis, dass das deutsche Gesundheitswesen durch eine Reihe von Missständen geprägt wird. Festzustellen sind die Unter-, Übersowie die Fehlversorgung der Patienten. Außerdem steht den im internationalen Vergleich relativ hohen Gesundheitsausgaben kein entsprechend hohes Versorgungsniveau gegenüber. Als weiterer Kritikpunkt ist die übermäßige Komplexität des Systems und der zugrunde liegenden Regelungen zu nennen. Als eine wesentliche Ursache für diese Missstände wurde die strikte sektorale Trennung des Versorgungsgeschehens ausgemacht und zwar sowohl hinsichtlich der Erbringung als auch hinsichtlich der Finanzierung der Leistungen in der gesetzlichen Krankenversicherung. Soweit Wettbewerb zwi- 
schen den gesetzlichen Krankenkassen möglich ist, findet dieser ferner im Wesentlichen über die unterschiedliche Höhe des Beitragssatzes und nicht - wie angestrebt - über die Qualität der Leistungserbringung statt.

Abhilfe soll die Einführung bzw. der Ausbau von Vertragswettbewerb schaffen, wobei hierunter ein wettbewerbliches Gesamtkonzept zur Verbesserung der Qualität und der Wirtschaftlichkeit der Versorgung der Versicherten zu verstehen ist. Wettbewerbliche Elemente im Gesundheitswesen haben zur Erreichung dieses Ziels zu dienen, ihre Existenz ist deshalb kein Selbstzweck. Erforderlich ist es hierfür, die bestehende Regulierung abzubauen und den Beteiligten größere Handlungsspielräume zuzugestehen. Damit werden wettbewerbliche Such- und Entdeckungsprozesse ausgelöst, die letztlich zur Behebung oder Reduzierung der oben genannten Defizite führen sollen. Um dies zu ermöglichen, wird den Krankenkassen im Konzept des Vertragswettbewerbs die Kompetenz zum Abschluss von Einzelverträgen mit Entscheidungsfreiheit bezüglich der Auswahl ihrer Vertragspartner, der Vergütungsformen sowie der Vertragsinhalte im Übrigen gegeben. Nicht zuletzt ist die Einbeziehung der Versicherten (etwa über Anreize) erforderlich, damit Vertragswettbewerb - als Wettbewerb um die Versicherten - gelingen kann.

\subsection{Kriterien für die Beurteilung der Neuregelungen zur integrierten Versorgung}

Ziel des Beitrags ist es, die jüngsten Neuregelungen zur integrierten Versorgung unter dem Gesichtspunkt zu beurteilen, inwieweit sie zur Verwirklichung des Vertragswettbewerbs beitragen (s. oben 1.). Eine solche Beurteilung ist nur anhand von operationalisierbaren Einzelkriterien möglich, die aus dem Konzept des Vertragswettbewerbs abzuleiten sind. Aufgrund der Merkmale des Vertragswettbewerbs wird gefragt, inwieweit die rechtlichen Änderungen der Vorschriften dazu führen, dass

- den Krankenkassen zusätzlicher Handlungsspielraum eingeräumt wird,

- die Grenzen zwischen den einzelnen Versorgungsbereichen abgebaut werden,

- die Komplexität der Regelungen und des Gesundheitssystems reduziert wird und

- der integrierten Versorgung insgesamt zur weiteren Verbreitung verholfen wird.

\section{Vertragswettbewerb zwischen Krankenkassen in der integrierten Versorgung}

\subsection{Verhältnis der Krankenkassen zu den Vertragspartnern}

Im Verhältnis der Krankenkassen zu den Vertragspartnern spielt zunächst die Auswahl der möglichen Vertragspartner eine Rolle. Sodann ist der Bereich der Finanzierung der integrierten Versorgung sowie der Vergütung der Vertragspartner bzw. der Verwendung der Anschubmittel zu betrachten. Schließlich wird auf Änderungen der Vorgaben für den Inhalt von Verträgen über integrierte Versorgung im Übrigen eingegangen.

\subsubsection{Vertragspartner der Krankenkassen}

Der Kreis der möglichen Beteiligten beim Abschluss von Verträgen der Krankenkassen in der integrierten Versorgung wurde durch die Einführung des § 77a SGB V erweitert. Danach können die Kassenärztlichen Vereinigungen und die Kassenärztlichen Bundesvereinigungen anders als bisher -Dienstleistungsgesellschaften gründen (§ 77a Abs. 1 SGB V), die gegenüber vertragsärztlichen Leistungserbringern die in $\$ 77$ a Abs. 2 SGB V näher bezeichneten Aufgaben erfüllen können. Dazu gehören z.B. die Beratung beim Abschluss von Versorgungsverträgen (Nr. 1) oder die Vertragsabwicklung bei derartigen Verträgen (Nr. 4). Die Dienstleistungsgesellschaften können also in vielerlei Hinsicht unterstützend beim Abschluss von Verträgen der integrierten Versorgung tätig werden und damit zu einer effizienteren Erledigung der gestiegenen Managementaufgaben in den flexibleren Vertragsstrukturen beitragen. ${ }^{4}$ Diese Änderung ist aufgrund des damit verbundenen Professionalitätsgewinns unter dem Gesichtspunkt der leichteren Verbreitung der integrierten Versorgung zu begrüßen. Was § 77a SGB V dagegen nicht enthält, ist die Kompetenz der Dienstleistungsgesellschaften zum Abschluss von Verträgen in der Eigenschaft als Vertragspartner. In der Gesetzesbegründung wird die Aufzählung der Aufgaben in § 77a Abs. 2 SGB V ausdrücklich als abschließend und die Dienstleistungsgesellschaften lediglich als „Ansprechpartner für den einzelnen Vertragsarzt" bezeichnet. ${ }^{5}$ Da die Dienstleistungsgesellschaften auch nicht in $§ 140 \mathrm{~b}$ Abs. 1 SGB V genannt werden, kommen diese nicht als Vertragspartner der Krankenkassen für den Abschluss von Verträgen zu integrierten Versorgung in Betracht. ${ }^{6}$

Neu eingeführt wurde die Befugnis der Krankenkassen gem. § 140b Abs. 1 Nr. 5 SGB V, auch mit Pflegekassen und mit zugelassenen Pflegeeinrichtungen als Vertragspartner auf der Grundlage des § 92b SGB XI Verträge über integrierte Versorgung abzuschließen. Auch diese Regelung ist positiv zu bewerten. Erstmals wird hierdurch nicht nur eine Integration des Versorgungsgeschehens über verschiedene Sektoren, sondern sogar über die Grenzen der Versicherungszweige Kranken- und Pflegeversicherung hinweg ermöglicht. Dafür besteht ein großes praktisches Bedürfnis. Gleichzeitig wird der Gestaltungsspielraum der Krankenkassen vergrößert. ${ }^{7}$

Neben dem Kreis der möglichen Vertragspartner ist für die Krankenkasse daneben von großer Bedeutung, an welches Verfahren sie sich ggf. bei der Auswahl der konkreten Vertragspartner halten muss. Fraglich sind in dem Zusammenhang die Auswirkungen des in § 69 S. 2 SGB V eingefügten Verweises auf die $\$ \S 19-21$ GWB, die entsprechend gelten sollen. ${ }^{8}$ Es handelt sich um eine eingeschränkte Rechtsgrundverweisung, aufgrund derer das Vorliegen eines Unternehmens im kartellrechtlichen Sinn nicht gesondert geprüft werden muss. In den Fällen, in 
denen von der Marktbeherrschung oder einer marktstarken Stellung der handelnden Krankenkasse(n) auszugehen ist, unterliegen diese zukünftig den Maßstäben der kartellrechtlichen Missbrauchskontrolle. Festzuhalten ist, dass lediglich ein kleiner Teil der Vorschriften des GWB und dieser auch nur sinngemäß anwendbar ist. ${ }^{9}$ Desweiteren bleiben die Sozialgerichte für die Entscheidung über eventuelle Verstöße zuständig ( $\$ 51$ Abs. 2 SGG). Für das Verfahren zur Auswahl von Vertragspartnern kann der Verweis bei Vorliegen der Voraussetzungen für die Krankenkasse bedeuten, dass zur Vermeidung des Vorwurfs der Kartellrechtswidrigkeit die Durchführung einer Ausschreibung angezeigt ist, die sich zwar nicht nach den strikten Frist- und Formvorgaben des Kartellvergaberechts richten, jedoch die Grundsätze der Transparenz, des Wettbewerbs sowie der Nichtdiskriminierung wahren muss.

Was die Anwendbarkeit des Kartellvergaberechts angeht, sei an dieser Stelle lediglich darauf hingewiesen, dass die Regelungen über die integrierte Versorgung keinen ausdrücklichen diesbezüglichen Anwendungsbefehl enthalten. Auch fehlt es an den Voraussetzungen der Anwendbarkeit des Kartellvergaberechts gem. §§ 98 f. GWB. Gesetzliche Krankenkassen sind keine öffentlichen Auftraggeber im Sinn des § 98 Nr. 2 GWB, da sie nicht durch staatliche Stellen finanziert werden, sondern durch die Versicherten und die Arbeitgeber. ${ }^{10}$ Sie unterliegen auch nicht der Aufsicht im Sinne des § 98 Nr. 2 GWB, für die die bloße Rechtsaufsicht nach herrschender Meinung nicht ausreichend ist. Die gesetzlichen Krankenkassen unterliegen jedoch gem. § 87 Abs. 1 SGB IV ausschließlich der Rechts- und nicht der Fachaufsicht. ${ }^{11}$ Darüberhinaus werden Verträge über integrierte Versorgung im Regelfall keine entgeltlichen Aufträge gem. § 99 Abs. 1 GWB sein, wobei jedoch die Gestaltung des einzelnen Vertrages ausschlaggebend ist. In der Regel erhält der Vertragspartner der Krankenkasse die Befugnis, seine Leistungen im Rahmen der integrierten Versorgung anzubieten und entsprechend abzurechnen, wobei er in vollem Umfang das wirtschaftliche Risiko des Gelingens der integrierten Versorgung trägt, da die Krankenkasse insbesondere aufgrund des Wahlrechts der Versicherten daran gehindert ist, den Leistungserbringern etwa bestimmte Fallzahlen zuzusichern. Aus diesem Grund wird häufig eine Konzessionssituation vorliegen, für die die Vorgaben des Vergaberechts nicht gelten. ${ }^{12}$

Schließlich ist es in Bezug auf die Auswahl der Vertragspartner der Krankenkasse mit dem Konzept des Vertragswettbewerbs unvereinbar, eine Pflicht zum Abschluss von Verträgen mit allen Interessierten anzunehmen. Ein Kontrahierungszwang der Kassen besteht insoweit nicht.

\subsubsection{Finanzierung der integrierten Versorgung und Verwendung der Anschubmittel}

In Bezug auf die Finanzierung der integrierten Versorgung ist einleitend allgemein darauf hinzuweisen, dass die Bereiche des Einbehalts der Anschubfinanzierung einerseits und der Verwendung der Anschubmittel andererseits gedanklich voneinander zu trennen sind. Diese folgen unterschiedlichen Regelungen und sind nicht notwendig miteinander verknüpft, etwa in dem Sinn, dass die Mittel nur in dem Sektor verwendet werden dürften, in dem sie einbehalten worden sind. Eine solche Einschränkung enthält das Gesetz nicht.

Mit dem Gesetz zur Änderung des Vertragsarztrechts und anderer Gesetze (VÄndG) ${ }^{13}$ wurde der Zeitraum, in dem die Krankenkassen zur Förderung der integrierten Versorgung Anschubmittel von den Gesamtvergütungen und den Krankenhausrechnungen einbehalten können, bis zum Ende des Jahres 2008 verlängert (§ 140d Abs. 1 S. 1 SGB V). Hierdurch wird Sicherheit für die bislang in die Etablierung der integrierten Versorgung getätigten Investitionen geschaffen, was die Weiterverbreitung der integrierten Versorgung stärkt. Der Wert der Verlängerung des Anschubzeitraums für die Kassen wird leider dadurch geringfügig eingeschränkt, dass diese erst relativ spät vorgenommen wurde und die Kassen deshalb nicht von vornherein für den längeren Zeitraum planen konnten.

Eine Erhöhung der Abzugsquote auf mehr als einen Prozentpunkt hat der Gesetzgeber dagegen abgelehnt. Das ist inkonsequent, denn seit dem 01.04.2007 sollen die Verträge zur integrierten Versorgung gem. § 140a Abs. 1 S. 2 SGB V eine bevölkerungsbezogene Flächendeckung der Versorgung ermöglichen. Wie eine flächendeckende Versorgung mit lediglich einem Prozent der Gesamtvergütungen und Krankenhausrechnungen finanziert werden soll, hat der Gesetzgeber nicht erläutert. Untauglich ist in diesem Zusammenhang der Verweis auf die Möglichkeit, den Finanzbedarf außerhalb der Anschubmittel zu decken und die Gesamtvergütungen gem. § 140d Abs. 2 SGB V zu bereinigen. Zurecht wurden die nicht praktikablen Vorgaben für die Bereinigung im Zuge der vorhergehenden Gesundheitsreform als eines der wesentlichen Hemmnisse für die Verbreitung der integrierten Versorgung angesehen und folglich die Anschubfinanzierung eingeführt.

Durch die Einfügung des neuen Satzes 2 1. HS. in $\S 140 d$ Abs. 1 SGB V wurden die Möglichkeiten der Verwendung der Anschubmittel eingeschränkt. Die nach § 140d Abs. 1 S. 1 SGB V einbehaltenen Mittel dürfen demnach nur noch für voll- oder teilstationäre und ambulante Leistungen der Krankenhäuser und für ambulante vertragsärztliche Leistungen verwendet werden. Dies gilt für die seit dem 01.04.2007 abgeschlossenen Verträge. Der Gesetzgeber begründet diese Beschränkung der Verwendungsmöglichkeiten damit, dass der vorher zulässige Einsatz der Mittel in allen Versorgungsbereichen zu einer Quersubventionierung geführt hätte. Diese sei zwar im Interesse eines unbürokratischen Starts der integrierten Versorgung gerechtfertigt gewesen, sei nun aber nicht mehr hinnehmbar. ${ }^{14}$ Positiv an diesen Äußerungen ist lediglich die beiläufig enthaltene Bestätigung, dass die Verwendung der Anschubmittel bei den bis zum 30.03.2007 abgeschlossenen Verträgen ohne Einschränkung möglich ist. Abgesehen hiervon ist die begrenzende Regelung des $§ 140 d$ Abs. 1 S. 2 1. HS. SGB V als Rückfall in sektorales Denken abzulehnen. 
Im 2. Halbsatz des $§ 140 d$ Abs. 1 S. 2 SGB V nimmt der Gesetzgeber die Aufwendungen für besondere Integrationsaufgaben von der gerade genannten Beschränkung aus. Als Beispiele für die besonderen Integrationsaufgaben werden in der Gesetzesbegründung die Koordinierung von Leistungen sowie das Casemanagement genannt. ${ }^{15}$ Gerade die Erledigung dieser Aufgaben ist für das Gelingen von integrierter Versorgung von entscheidender Bedeutung, weshalb es zu begrüßen ist, dass die Verwendung von Mitteln aus der Anschubfinanzierung für diese Aufgaben - jetzt durch eine ausdrückliche gesetzliche Regelung - für zulässig erklärt wird. Die Rechtsprechung wird zeigen, wie der Begriff der Aufwendungen für besondere Integrationsaufgaben im Einzelnen auszulegen ist.

Der neu ins Pflegeversicherungsrecht eingefügte $\S 92 \mathrm{~b}$ SGB XI, auf dessen Grundlage der Abschluss von integrierten Versorgungsverträgen auch für Pflegekassen möglich wird, ${ }^{16}$ enthält zwei Restriktionen im Bereich der Finanzierung. Gemäß § 92b Abs. 2 S. 3 SGB XI dürfen in den Pflegevergütungen keine Aufwendungen berücksichtigt werden, die nicht der Finanzierungszuständigkeit der sozialen Pflegeversicherung unterliegen. Mit dieser Regelung soll vermieden werden, dass es zu Kostenverlagerungen zulasten der Pflegeversicherung kommt. ${ }^{17} \mathrm{Au}-$ ßerdem müssen Mehraufwendungen, die den Pflegeeinrichtungen durch die integrierte Versorgung entstehen, gem. § 92b Abs. 2 S. 4 SGB XI durch Zuschläge zu den Pflegevergütungen finanziert werden. Eine Anschubfinanzierung ist im SGB XI nicht vorgesehen. Auch wenn die gesetzgeberischen Motive für diese Vorschriften nachvollziehbar sind, so ermöglichen die getroffenen Regelungen doch nicht den Abbau von Sektorabschottungen und sind deshalb abzulehnen. Der Aufbau von integrierter Versorgung über die Grenzen der Versicherungszweige hinweg wird mit diesen Vorgaben schwerfallen.

\subsubsection{Regelungen zum Vertragsinhalt im Übrigen}

Das GKV-WSG hat die rechtlichen Vorgaben für den Vertragsinhalt der integrierten Versorgung in Bezug auf die Erbringung von hochspezialisierten Leistungen sowie auf die Arzneimittelversorgung geändert.

Die Krankenhäuser sind jetzt - unabhängig davon, ob ein anderer Vertragspartner in der integrierten Versorgung über den entsprechenden Zulassungsstatus verfügt - im Rahmen einer integrierten Versorgung zur ambulanten Behandlung der im Katalog nach $§ 116 b$ Abs. 3 SGB V genannten hochspezialisierten Leistungen, seltenen Erkrankungen und Erkrankungen mit besonderen Behandlungsverläufen berechtigt ( $\$ 140 b$ Abs. 4 S. 4 SGB V). Diese Neuregelung birgt Chancen und Risiken. Es besteht die Chance des Vertragsabschlusses mit hochqualifizierten Einrichtungen. Andererseits kann es bei fehlenden Steuerungsmöglichkeiten der Kasse zu Doppelfinanzierungen kommen.

§ 140a Abs. 1 S. 5 SGB V sieht seit dem GKV-WSG vor, dass die für die ambulante Behandlung im Rahmen der integrierten Versorgung erforderliche Arzneimittelversor- gung durch Arzneimittelrabattverträge gem. § 130a Abs. 8 SGB V erfolgen soll. Die Vorschrift ermöglicht die Steuerung von Versicherten zu Versorgungen auf der Grundlage der Rabattverträge. Durch diese Vorschrift werden quasi zwei Möglichkeiten zum Abschluss von Einzelverträgen kombiniert. Die Regelung stellt einen zusätzlichen Anreiz für pharmazeutische Unternehmen zur Beteiligung an Rabattverträgen gem. § 130a Abs. 8 SGB V dar. Auch unter dem Gesichtspunkt der Wirtschaftlichkeit der Versorgung handelt es sich um eine positiv zu bewertende Änderung.

Im Gesetzentwurf war ursprünglich eine zusätzliche inhaltliche Vorgabe für den Abschluss von integrierten Versorgungsverträgen vorgesehen. § 140d Abs. 1 S. 2 SGB V sah in der damaligen Fassung vor, dass die Anschubmittel ausschließlich für Verträge verwendet werden dürfen, die eine bevölkerungsbezogene Flächendeckung der Versorgung zum Gegenstand haben. ${ }^{18}$ Diese Einschränkung hätte die wettbewerblichen Such- und Entdeckungsprozesse behindert, die die integrierte Versorgung in Gang setzen soll. Mit Hinblick darauf, dass auch sinnvolle Projekte ohne Bevölkerungsbezug mit der finanziellen Unterstützung der Anschubfinanzierung möglich bleiben sollen, wurde diese Änderung zurecht durch den Gesundheitsausschuss gestrichen. ${ }^{19}$

\subsection{Verhältnis zwischen Krankenkassen und Versicherten}

Für das Verhältnis zwischen den Krankenkassen und den Versicherten in der integrierten Versorgung ist maßgeblich, wie die Anreize für die Teilnahme der Versicherten an der integrierten Versorgung ausgestaltet sind. Die Teilnahme an der integrierten Versorgung ist weiterhin freiwillig. Die Einschreibung der Versicherten empfiehlt sich zwar aus praktischen Gründen, die Entscheidungsfreiheit des Versicherten bleibt davon jedoch unangetastet.

Nach altem Recht konnte die Krankenkasse bei Teilnehmern an einer integrierten Versorgung in ihrer Satzung die Zuzahlungen ermäßigen (\$ 65a Abs. 2 S. 1 SGB $\mathrm{V}$ a.F.). Ob sie eine entsprechende Satzungsregelung treffen wollte oder nicht, oblag also ihrer eigenen freien Entscheidung. Seit dem In-Kraft-Treten des GKV-WSG trifft die Krankenkassen gem. § 53 Abs. 3 SGB V die Pflicht, für Versicherte, die an einer integrierten Versorgung teilnehmen, in ihrer Satzung besondere Tarife anzubieten. Sie kann für diese Versicherten eine Prämienzahlung oder Zuzahlungsermäßigungen vorsehen. Durch die neu eingeführte Pflicht der Krankenkasse, einen besonderen Tarif anzubieten, werden die Gestaltungsspielräume der Kasse unnötig eingeschränkt. Es sollte ihr überlassen sein, ob sie Einführung besonderer Tarife für sinnvoll hält oder nicht.

\subsection{Weitere Einflussfaktoren}

Neben den Vorschriften unmittelbar über die integrierte Versorgung beeinflussen weitere Faktoren das Ge- 
lingen des Vertragswettbewerbs auch im Bereich der integrierten Versorgung. Dies ergibt sich schon daraus, dass Vertragswettbewerb ein Gesamtkonzept darstellt. Erforderlich ist insgesamt eine solidarische Wettbewerbsordnung. Dazu gehört wesentlich eine angemessene Ausgestaltung des morbiditätsorientierten Risikostrukturausgleichs. Von Bedeutung ist auch im Hinblick auf die integrierte Versorgung die ab dem 01.01.2009 bestehende Pflicht der Krankenkassen, ggf. einen kassenindividuellen Zusatzbeitrag zu erheben. ${ }^{20}$ Hierdurch wird ein erheblicher Anreiz in Richtung Beitragswettbewerb auf die Kassen ausgeübt. Die Entwicklung und Etablierung neuer Versorgungsformen erfordern Aufwand und Investitionen. Es kann nicht ausgeschlossen werden, dass eine Krankenkasse vor dem Hintergrund eines eventuell zu erhebenden Zusatzbeitrags, der sie im Wettbewerb mit konkurrierenden Kassen schlechterstellt, von an sich sozialpolitisch gewünschten Investitionen in die neuen Versorgungsformen Abstand nimmt. Nicht zuletzt stellen auch die steuerrechtlichen Rahmenbedingungen der integrierten Versorgung einen nicht unerheblichen Einflussfaktor dar.

\section{Zusammenfassende Bewertung}

Die Änderungen an den Rechtsvorschriften der integrierten Versorgung durch das VÄndG und das GKVWSG führen nicht zu einem grundlegend neuen Rechtsrahmen. Dies war auch nicht angezeigt, denn vieles hat sich bewährt. Der wesentlichste Bedarf war es, mehr Zeit für die Etablierung der integrierten Versorgung zu haben. Dieses größere Zeitfenster haben die Beteiligten erhalten. Nahezu ebenso wichtig wäre es aber gewesen, die zur Verfügung stehenden Mittel durch eine höhere Abzugsquote bei der Anschubfinanzierung zu erhöhen, was leider unterblieben ist

Einige einzelne Änderungen sind im Hinblick auf die Verwirklichung des Vertragswettbewerbs nicht förderlich. $\mathrm{Zu}$ nennen sind etwa nochmal die beschränkten Verwendungsmöglichkeiten der Anschubmittel nach § 140d Abs. 1 S. 2 SGB V, die als Rückfall in sektorales Denken zu werten sind, die ungelösten Bereinigungsprobleme oder die Defizite bei der Einbeziehung der Pflegeversicherung. Insgesamt erfüllen die Neuerungen - wie der Anspruch war - die Unterstützungsfunktion in Bezug auf die Wei- terentwicklung der IV, deren weitere Umsetzung in der Verantwortung der Beteiligten liegt.

\section{Fußnoten}

1 BR-Drucks. 755/06 zu Art. 1 Nr. 119 Buchstabe a) des Gesetzentwurfs.

2 BGBl 2007 I S. 378

3 Ebsen, Ingwer/Greß, Stefan/Jacobs, Klaus/Szecsenyi, Joachim/Wasem, Jürgen, Vertragswettbewerb in der gesetzlichen Krankenversicherung zur Verbesserung von Qualität und Wirtschaftlichkeit der Gesundheitsversorgung, 2003; Cassel, Dieter/Ebsen, Ingwer/Greß, Stefan/ Jacobs, Klaus/Schulze, Sabine/Wasem, Jürgen, Weiterentwicklung des Vertragswettbewerbs in der gesetzlichen Krankenversicherung. Vorschläge für kurzfristig umsetzbare Reformschritte, 2006

4 Vgl. hierzu die Begründung zum Gesetzentwurf zu Art. 1 Nr. 49; BRDrucks. 755/06 S. 322.

5 Wie vorhergehende Fußnote.

6 Der Gesetzgeber hat es auch beim schon bisher bestehenden Ausschlus der Kassenärztlichen Vereinigungen aus dem Kreis der möglichen Vertragspartner der Kassen belassen.

7 Siehe aber noch unten 3.1.2. am Ende.

$8 \mathrm{Zu}$ den Ausnahmen von der entsprechenden Anwendbarkeit vgl. § 69 S. 2 2. HS SGB V.

9 Nicht anwendbar ist etwa $\S 1$ GWB, weshalb der bloße Zusammenschluss von Krankenkassen zwecks gemeinschaftlichen Vertragsabschlusses zulässig ist.

10 Die Höhe des Bundeszuschusses zu den Ausgaben der gesetzlichen Krankenkassen erreichen nicht annähernd die relevante Größe einer überwiegenden staatlichen Finanzierung.

11 Vgl. Beschluss des BayObLG v. 24.05.2004 (Verg 6/04).

12 S. EuGH v. 13.10.2005 (C-458/03).

13 BGBl 2006 I S. 3439.

14 Vgl. die Begründung zu Art. 1 Nr. 121 Buchst. a) aa), BR-Drucks. 755/06 S. 415.

15 Wie vorhergehende Fußnote.

16 S. oben 3.1.1.

17 So die Begründung zu Art. 8 Nr. 40, BR-Drucks. 755/06 S. 514.

18 Eine solche bevölkerungsbezogene Flächendeckung der Versorgung hätte nach der Gesetzesbegründung insbesondere dann vorgelegen, wenn „entweder in einer größeren Region (z.B. mehrere Stadt- oder Landkreise) die Behandlung einer versorgungsrelevanten Volkskrankheit (z.B. Diabetes, Schlaganfallprävention oder Bandscheibenerkrankungen) umfassend in einer integrierten Versorgung angeboten wird oder in einer auch kleineren Region das gesamte oder ein Großteil des Krankheitsgeschehens der Versicherten in einer integrierten Versorgung ermöglicht wird." Vgl. Begründung zu Art. 1 Nr. 119, BR-Drucks. 755/06 S. 413. Trotz dieser Erläuterungen hätte es in der Praxis erhebliche Auslegungsschwierigkeiten gegeben.

19 Vgl. BT-Drucks. 16/4247 S. 49.

$20 \S 242$ SGB V in der ab 2009 geltenden Fassung, vgl. Art. 1 Nr. 161 GKV-WSG, BGB1. 2007 I S. 378. 